\title{
Recommendations of the Group of Experts of the Polish Society of Gynecologists and Obstetricians regarding abnormal uterine bleeding in adolescents
}

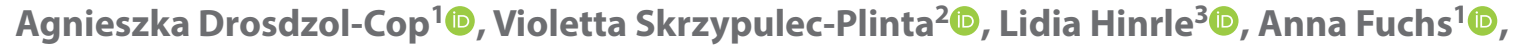 \\ Iwona Joanna Czech ${ }^{4}\left(\mathbb{0}\right.$, Grazyna Jarzabek-Bielecka ${ }^{5}$ (i) \\ ${ }^{1}$ Chair and Department of Gynecology, Obstetrics and Oncological Gynecology, Medical University of Silesia in Katowice, Poland \\ ${ }^{2}$ Chair of Woman's Health, Medical University of Silesia in Katowice, Poland \\ ${ }^{3} 7^{\text {st }}$ Department and Clinic of Gynaecology and Obstetrics, Wroclaw Medical University, Poland \\ ${ }^{4}$ Department of Woman's Health, School of Health Sciences in Katowice, Medical University of Silesia, Katowice, Poland \\ ${ }^{5}$ Division of Gynecology, Department of Perinatology and Gynecology, Poznan University of Medical Sciences, Poland
}

\section{INTRODUCTION}

The recommendations present the current knowledge and procedures, which can be modified and changed in some cases, after careful analysis of a given clinical situation, which in the future may become the basis for their modification and updating.

\section{Menstrual cycle in adolescents}

Normal menstrual cycles in adolescents last 21-45 days, with 2-7 days of menstrual bleeding and $20-80 \mathrm{~mL}$ of menstrual blood loss. During the first year after menarche, the mean duration of the menstrual cycle is 32.2 days. In the majority of teenagers, in the first 2-3 years after the menarche, menstrual cycles are irregular, which is caused by anovulation and immaturity of the hypothalamic-pituitary-ovarian system [1, 2].

Anovulatory menstrual cycles are present typically during first years of menarche. The rate of ovulation depends on the period of time from the first menstruation and the age of menarche. Earlier menarche age correlates with faster onset of ovulatory cycles. Some girls who had menarche before the age of 12 have ovulation in $50 \%$ of their menstrual cycles in the first year, while at the point of later menarche, the ovulatory menstrual cycle stabilizes even after 8-12 years. By the $3^{\text {rd }}$ year after menarche, $60-80 \%$ of menstrual cycles are normalized (mean duration 21-34 days). It is also believed that the time needed to normalize the menstrual cycle may extend up to 6 years after menarche [1, 2].

\section{Abnormal uterine bleeding - definition, epidemiology, etiology}

In adolescents, heavy menstrual bleeding (HMB) is most often caused by anovulatory irregular menstrual cycles and can occur in two forms:

- as abnormal uterine bleeding (AUB), the most common clinical picture, with irregularity in the timing of bleeding, cycle length and the volume of menstrual blood lost;

- as episodes of amenorrhea for an average of 3 months, followed by 1-day bleeding in the next month (non-cyclic or insufficient exfoliation of the functional layer of the endometrium, which is thickened, resulting in amenorrhea for the next few months, during which there is a slow growth endometrium) - a less common clinical picture [1-3].

Less frequently, heavy menstrual bleeding in adolescents take the form of functional anovulatory uterine bleeding (predominantly at the beginning of adolescence) [1-3].

The most common cause of abnormal bleeding from the genital tract (AUB) in adolescence is juvenile bleeding, defined as profuse bleeding with clots lasting more than 10 days, often leading to anemia, not associated with any organic pathology of the reproductive organ and systemic diseases. The menstrual blood loss usually exceeds $80 \mathrm{~mL}$. Such bleeding episodes that are not related to the menstrual cycle can occur up to 5 years after the menarche; they are characterized by various bleeding times and intervals between episodes, as well as different degrees of bleeding: from moderate to heavy. It is a very common form of menstrual disorders in adolescence, usually of a functional nature. 
The main cause of juvenile bleeding in girls are anovulatory cycles, physiologically lasting up to 2-3 years after the menarche, caused by immaturity of the hypothalamic-pituitary-ovary system. However, the etiopathogenesis of juvenile bleeding has not been clearly established - most adolescents do not have ovulation in the first years after the menarche, but only some girls experience abnormal heavy vaginal bleeding [1-3].

Juvenile bleeding occurs in about $12-37 \%$ of adolescent girls. In $20 \%$ of cases, such episodes are observed already during the first menstruation, and in $80 \%$ they occur in the period from one to two years after the menarche. Such heavy menstrual bleeding is often described by girls as: the presence of clots in the menstrual blood $\geq 2.5 \mathrm{~cm}$ in diameter, the need to change the pads/tampons more often than every hour, the pads / tampons become soaked after $1 \mathrm{~h}$ for the next 2-3 hours, the use of "double protection" (a pad and a tampon or 2 pads in total), menstrual bleeding, "gushing" sensations of blood, frequent incidents of leaking pads, and iron deficiency anemia may occur [1-3].

Abnormal vaginal bleeding in girls in the form of juvenile bleeding does not have organic origin but is caused by anovulation (90\%) or luteal failure (10\%). Post-menstrual cycles are usually anovulatory, more or less irregular, and with varying degrees of bleeding. During this period, pulse secretion of gonadotrophins also begins during the day and is regulated until the frequency and amplitude of the pulses reach the value characteristic of ovulatory cycles. The mucosa of the uterine cavity proliferates upon exposure to estrogens in the follicular phase (normal FSH pulses), and the lack of progesterone in the luteal phase (no LH pulse in the middle of the cycle) does not adequately stabilize the endometrium which does not undergo secretory transformation. The mucosa peels off and there is profuse, unrelated to the menstrual cycle, sometimes difficult to control bleeding. The endometrium is atrophied as manifested by continual vaginal bleeding. The above fact indicate that the use of only progestins (cyclically, in the luteal phase) in the treatment of adolescent bleeding in girls often does not bring the expected results and may lead to the need for curettage of the uterine cavity [1-5].

Excessive, abnormal, prolonged bleeding during adolescence may also occur in girls in other clinical conditions related to anovulation, such as: eating disorders, weight changes, chronic systemic diseases, intense emotional stress, excessive physical activity related to playing sports, drug abuse, ovarian tumors, endocrine disorders, and polycystic ovary syndrome (PCOS) (20-30\% of PCOS patients have pre-existing AUB) [1-5].

\section{Differential diagnosis}

Although the most common type of abnormal bleeding from the genital tract in adolescent girls are juvenile bleed- ing (non-ovulatory, functional), which accounts for 45-97\% of all causes, in each case of prolonged bleeding a careful differential diagnosis should be performed and any possible etiology of the symptoms should be ruled out. The causes of AUB in girls include [1-8]:

\section{- juvenile bleeding (non-ovulatory, functional)} - 45-97\%;

- pregnancy-related disorders (miscarriage, ectopic pregnancy, gestational trophoblastic disease);

- sexually transmitted infections (STI; pelvic inflammatory syndrome, endometritis, cervicitis, vaginitis, Neissesia gonorrhoeae and Chlamydia trachomatis infection);

- coagulation disorders (thrombocytopenia, von Willebrand's disease, congenital disorders of coagulation factors, platelets, leukemia, aplastic anemia, liver failure) - 3-19\%;

- endocrine disorders (hypothyroidism and hyperthyroidism, hyperprolactinaemia, PCOS, adrenal gland disease, ovarian failure in Turner syndrome, after chemotherapy/radiotherapy);

- cervical diseases (polyps, inflammation, hemangiomas, neoplastic changes - very rarely);

- vaginal diseases (inflammation, neoplastic changes - very rarely);

- endometrial pathologies (congenital malformations, submucosal fibroids, polyps, bleeding associated with the use of hormonal contraceptives or IUD, neoplastic changes - very rarely);

- pathologies of appendages (cystic, neoplastic changes);

- endometriosis;

- injuries to the reproductive organ (sexual abuse);

- presence of a foreign body in the vagina (most often an unremoved, forgotten tampon);

- systemic diseases (diabetes, kidney diseases, systemic lupus);

- medications used (hormonal contraception, androgens, spironolactone, anticoagulants, antipsychotics).

\section{The diagnosis of AUB}

Important elements in the diagnostic process of juvenile bleeding are: clinical history, physical examination, basic laboratory tests and imaging tests (in selected cases). The clinical history should cover the following issues [1-8]:

\section{Gynecological history}

- menarche - the older the age of the first menstruation, the longer the period of anovulatory cycles, a heavy first menstruation may suggest congenital disorders of the coagulation system;

- length and regularity of menstrual cycles (based on the menstrual calendar and Menstrual Bleeding Questionnaire - MBQ), presence of dysmenorrhea [6];

- characteristics of menstrual bleeding - (based on the Menstrual Bleeding Questionnaire - MBQ) date of 
the last menstruation, length, abundance, presence of blood clots during the day and night, average number of pads/tampons used per day (it is estimated that the consumption of more than 3 soaked sanitary napkins) or 5-6 saturated, normal-size tampons per day for at least 3 days is equivalent to blood loss of more than $80 \mathrm{~mL}$ ) [6];

- episodes of galactorrhea, discharge from the nipples;

- STI risk factors (early age of sexual initiation, high risk behavior, multiple sexual partners, not using condoms, having intercourse during menstrual bleeding) and their history;

- date of last sexual contact, forms of contraception used;

- sexual abuse (may be related to a genital trauma).

\section{Medical history}

- presence of systemic diseases (coagulation disorders, diabetes, kidney and liver diseases, gastroenterological disorders);

- prone to bruising, bruising, nosebleeds or gums;

- previous surgical procedures;

- medications used (hormonal contraceptives, anticoagulants, psychiatric drugs);

- the presence of symptoms of hyperandrogenism (acne, hirsutism, acanthosis nigricans);

- exposure to stress (psychological factors);

- changes in body weight (diet, weight loss, increased physical activity).

\section{Family history}

- a family history of heavy menstrual bleeding (risk of von Willebrand disease);

- the presence of coagulation disorders;

- autoimmune diseases;

- endocrine disorders;

- neoplastic diseases.

\section{Physical examination}

Next element of the juvenile bleeding diagnostic algorithm is the physical examination, which should start with a careful assessment of the girl's general and emotional condition. Then, a typical physical examination should be performed, paying particular attention to [1-8]:

- height, weight, BMI, body build, nutritional status, fat distribution (excluding Cushing's syndrome, Turner syndrome);

- measurement of blood pressure (lying down and standing) and heart rate (excluding cardiovascular disorders as a consequence of severe anemia);

- presence of headaches, visual disturbances;

- the presence of symptoms of hyperandrogenism;

- presence of symptoms typical for coagulation disorders (bruises, ecchymosis);

- assessment of the thyroid gland (nodules, enlargement);

- assessment of the breast glands (galactorrhea);
- evaluation of sexual maturation - Tanner scale (pubarche, thelarche, adrenarche).

\section{Gynecological examination}

Gynecological examination in sexually inactive girls rarely requires examination with a speculum. It is enough to perform an internal examination with one finger to assess the palpation of the vaginal part of the cervix and exclude the presence of a foreign body in the vagina. It is also possible to supplement such a procedure with a transrectal examination, carefully examining the appendages area (exclusion of nodular changes). In selected clinical cases (massive vaginal bleeding, suspected foreign body or vaginal damage), even in sexually inactive girls, a complete gynecological examination under general anesthesia should be performed [1-8].

In sexually active girls, a full gynecological examination is recommended. Assess the external genitalia (the size of the clitoris), and in the speculum: the cervix and vaginal walls (exclusion of STI, foreign body, trauma, determination of the bleeding site), and at the same time take a swab to determine bacteriological culture (for Neissesia gonorrhoeae and Chlamydia trachomatis); in the combined two-handed examination - determine the correctness of the uterine body and appendages (excluding palpation, nodular changes). Sometimes there is a need for a gynecological examination under general anesthesia, even in sexually active girls [1-8].

As a supplement to the AUB diagnostic process, it is recommended to perform an ultrasound examination of the smaller pelvis (transabdominal transducer with a full bladder, rectal or vaginal — in sexually active girls), especially in the case when gynecological examination shows an adnexal lesion, suspected developmental defects of the reproductive organ or when gynecological examination cannot be performed due to profuse bleeding. In individual indications, other imaging tests can also be performed (computed tomography, magnetic resonance imaging) [1-8].

\section{Laboratory tests}

The routine laboratory tests in the diagnosis of adolescent bleeding in girls include [3-5, 7-8]:

- pregnancy test (serum $\beta$ HCG level, alternatively urine test) - pregnancy exclusion;

- complete blood count (with level of platelets, reticulocytes);

- glucose level;

- assessment of the coagulation system (prothrombin time - PT, activated partial thromboplastin time - APTT, bleeding time, activity of selected coagulation factors); PT and APTT will always be abnormal even in mild coagulation disorders; with NSAIDs, the bleeding 
time should be assessed $24-48$ hours after discontinuing NSAID;

- von Willebrand factor level assessment (to be performed a few weeks after discontinuation of estrogen therapy, which overstates the actual factor level) - approximately $24 \%$ of girls with chronic HMB have von Willebrand disease;

- blood group (blood group 0 is associated with a lower level of von Willebrand factor).

In selected clinical cases, laboratory diagnostics should be extended to hormonal tests [3-5, 7-8]:

- TSH, fT4, fT3 (exclude thyroid disorders);

- prolactin (may be slightly elevated after examination of the mammary glands, level $>100 \mathrm{ng} / \mathrm{mL}$ suggests pituitary adenoma);

- serum androgen levels (total and free testosterone, DHEAS, 17-OH-progesterone, androstendione), especially with symptoms of hyperandrogenism;

- LH and FSH (assessment of the functions of the pituitary gland and ovaries).

The American College of Obstetricians and Gynecologists (ACOG) recommends routine coagulation tests for AUB in girls who are under 18 years of age and have symptoms of abnormal uterine bleeding, especially if their $\mathrm{Hb}$ level is less than $10 \mathrm{~g} / \mathrm{dL}$. At the same time, these patients should be screened for anemia. In addition, screening for disorders of the coagulation system should be performed in girls in the case of: menstruation lasting $\geq 7$ days with heavy bleeding interfering daily activities, history of treatment of anemia, family history of coagulation disorders, excessive bleeding after tooth extraction or surgery [8] .

\section{The treatment of AUB}

Treatment of abnormal uterine bleeding in girls depends on the etiology, severity of the bleeding and the degree of maturity of the hypothalamic-pituitary-ovarian system. The principle of hormonal treatment of juvenile bleeding is estrogen supplementation to heal the bleeding sites in the atrophic endometrium and stimulate its proliferation and progestogens (in the luteal phase) to stabilize the uterine mucosa and regulate the menstrual cycle [1-9].

\section{Mild AUB}

Mild AUB is a prolonged period of menstrual bleeding or a shortened menstrual cycle lasting from at least 2 months; menstrual blood loss is moderate and the $\mathrm{Hb}$ level is $>12 \mathrm{~g} / \mathrm{dL}$ [1-9].

The therapeutic management of AUB of mild severity is preservative (proper diet, lifestyle). It is recommended to keep a menstrual calendar in order to closely monitor the following periods. Individually, according to indications, you can also use: vitamins and iron (preventing ane- mia), antifibrinolytic drugs that reduce menstrual blood loss by about $40-50 \%$ (aminocaproic acid $20 \mathrm{mg} / \mathrm{kg} /$ day in 2-3 doses, tranexamic acid 1-1.5 g orally 3-4 times/day up to 5 days during menstruation), non-steroidal anti-inflammatory drugs (recommended: mefenamic acid $250 \mathrm{mg}$ 2-4 times/day, naproxen 250-500 mg 2-4 times/day, ibuprofen 600-1200 mg/day, flurbiprofen, diclofenac, indomethacin), which reduce the amount of bleeding [1-9].

In the case of mild juvenile bleeding, follow-up is recommended after 3 months or earlier, when the applied treatment does not bring the expected results [1-9].

\section{Moderate AUB}

Moderate AUB is defined as prolonged and /or heavy menstrual bleeding (greater than 7 days) or shortening of the menstrual cycle with frequent periods (every 7-21 days); the loss of menstrual blood is moderate, and $\mathrm{Hb}$ levels indicate mild anemia ( $\mathrm{Hb}$ within 10-12 g/dL) [1-9].

Estrogen-progestogen (E-P) therapy, oral contraceptives (OCs), or progestogen therapy alone can be used to treat moderate AUB.E-P therapy is recommended when the bleeding persists for a long time and the thickness of the endometrium on ultrasound examination does not exceed $5 \mathrm{~mm}$. The following treatment regimen is proposed [1-9]:

\section{E-P therapy (initial stage):}

- estrogen orally $2 \times 2 \mathrm{mg}$ for 20 days;

- from day 10 , additionally include: dydrogesterone $20 \mathrm{mg} / \mathrm{d}$ or micronized progesterone; $100-150 \mathrm{mg} / \mathrm{d}$ for the next 10-14 days;

\section{E-P therapy (cycle 2):}

- oral estrogen $2 \times 2 \mathrm{mg}$ from the $5^{\text {th }}$ day of the cycle for 20 days;

- from the $15^{\text {th }}$ day of the cycle, additionally include: dydrogesterone $20 \mathrm{mg} / \mathrm{d}$ or micronized progesterone 100-150 mg/d for the next 10 days;

\section{E-P therapy (cycle 3 and 4):}

- oral estrogen $1 \times 2 \mathrm{mg}$ from the $5^{\text {th }}$ day of the cycle for 20 days;

- from the $15^{\text {th }}$ day of the cycle, additionally include: dydrogesterone $20 \mathrm{mg} / \mathrm{d}$ or micronized progesterone $100-150 \mathrm{mg} / \mathrm{d}$ for the next 10 days;

\section{E-P therapy (cycle 5 and 6):}

- oral estrogen $1 \times 1 \mathrm{mg}$ from the $5^{\text {th }}$ day of the cycle for 20 days;

- from the $15^{\text {th }}$ day of the cycle, additionally include: dydrogesterone $20 \mathrm{mg} / \mathrm{d}$ or micronized progesterone $100-150 \mathrm{mg} / \mathrm{d}$ for the next 10 days.

An alternative to E-P therapy (according to ACOG standards) is oral combined hormonal contraception. Good results are obtained with the use of OCs containing 30-35 $\mu$ g ethinylestradiol or natural estradiol and a progestogen ensuring adequate cycle control and endometrial stabilization (rec- 
ommended: norgestrel $0.3 \mathrm{mg}$ or levonorgestrel $0.15 \mathrm{mg}$ ). OCs containing desogestrel, norgestimate, dydrogesterone and dienogest also have a beneficial therapeutic effect. In case of moderate bleeding and mild anemia, it is recommended to use 1 tablet/day for 21 days [10].

When bleeding and anemia are more severe, the recommended dosage is:

- 1 pill 2 times/day for 3-4 days, until the bleeding stops completely;

- then: 1 pill/day until the end of the 21-day treatment cycle.

If bleeding returns during the above therapy, the dose of OCs should be increased to 2 tablets/day until the end of the 21-day treatment cycle.

Only in the case of massive bleeding and significant anemia is the following treatment regimen exceptionally proposed [10]:

- 1 pill 4 times/day for 2-4 days;

- 1 pill 3 times/day for 3 days;

- 1 pill 2 times/day for 2 weeks.

When using such high doses of OCs, due to side effects, it is often necessary to start antiemetics (recommended 2 hours before each dose of OCs). OCs should be used in the $21+7$ scheme, but if the withdrawal bleeding is too heavy, the interval between packages should be shortened to 3-4 days. In order to obtain the best cycle control, it is recommended to extend the OCs therapy ( 1 tablet/day) to 3-6 menstrual cycles [10].

If there are contraindications to the inclusion of estrogens or OCs, or there is poor tolerance of the above regimens, and as a continuation of EP or OCs therapy, it is recommended to use only progestagens - cyclically in the second phase of the menstrual cycle (for 10-12 days when the bleeding is moderate or 12-14 days when the bleeding is moderate, prolonged and abundant). Recommended progestogens, well stabilizing the endometrium in adolescents, are MPA (10-20 mg/day), 19-nortestosterone derivatives: NETA (5-10 mg/day) and lynesterol (5-10 mg/day) and dydrogesterone (10-20 mg/day). Alternatively, one can administer progesterone (100-200 mg) intramuscularly once, followed by a 10-14-day substitution with oral progestogen. The use of cyclic progestogenic therapy should be continued for the next 3-6 months [9].

There are also clinical reports showing a beneficial effect of the intrauterine hormonal contraceptive system (releasing levonorgestrel) and long-acting $\mathrm{GnRH}$ analogues (used for the longest period of 6 months) in the treatment of moderate and severe adolescent bleeding [11].

Additionally, in moderate AUB, according to individual indications, the following can be used: vitamins and iron preparations (correction of anemia), antifibrinolytics, NSAIDs and possibly antibiotic therapy [1-9].

\section{Severe AUB}

Severe AUB is defined as profuse, prolonged vaginal bleeding associated with a disturbed menstrual cycle; menstrual blood loss is significant and $\mathrm{Hb}$ levels indicate significant anemia ( $\mathrm{Hb}$ levels $<10 \mathrm{~g} / \mathrm{dL}$ ). Symptoms of increasing anemia and haemodynamic failure are often observed, and in most cases hospitalization is indicated (especially when $\mathrm{Hb}<7 \mathrm{~g} / \mathrm{dL}$ ).

In the treatment of severe AUB $(\mathrm{Hb} 8-10 \mathrm{~g} / \mathrm{dL})$ and haemodynamic stability, it is recommended to use E-P therapy in the following scheme [1-9]:

\section{E-P therapy (initial stage):}

- oral estrogen 2-4 mg every 6 hours until bleeding stops or significantly reduces, then:

- estrogen orally $2 \times 2 \mathrm{mg}$ until the end of the 20-day treatment cycle;

- from day 10, additionally include: dydrogesterone $20 \mathrm{mg} / \mathrm{d}$ or micronized progesterone $100-150 \mathrm{mg} / \mathrm{d}$ for the next 10-14 days;

\section{E-P therapy (cycle 2):}

- oral estrogen $2 \times 2 \mathrm{mg}$ from the $5^{\text {th }}$ day of the cycle for 20 days;

- from the $15^{\text {th }}$ day of the cycle, additionally include: dydrogesterone $20 \mathrm{mg} / \mathrm{d}$ or micronized progesterone $100-150 \mathrm{mg} / \mathrm{d}$ for the next 10 days;

\section{E-P therapy (cycle 3 and 4):}

- oral estrogen $1 \times 2 \mathrm{mg}$ from the $5^{\text {th }}$ day of the cycle for 20 days;

- from the $15^{\text {th }}$ day of the cycle, additionally include: dydrogesterone $20 \mathrm{mg} / \mathrm{d}$ or micronized progesterone $100-150 \mathrm{mg} / \mathrm{d}$ for the next 10 days;

\section{E-P therapy (cycle 5 and 6):}

- oral estrogen $1 \times 1 \mathrm{mg}$ from the $5^{\text {th }}$ day of the cycle for 20 days;

- from the $15^{\text {th }}$ day of the cycle, additionally include: dydrogesterone $20 \mathrm{mg} / \mathrm{d}$ or micronized progesterone $100-150 \mathrm{mg} / \mathrm{d}$ for the next 10 days.

Alternatively (ACOG guidelines) in the treatment of severe $A U B(\mathrm{Hb} 8-10 \mathrm{~g} / \mathrm{dL})$ and ineffectiveness of E-P therapy, the use of OCs (containing 30-50 $\mu \mathrm{g}$ ethinylestradiol and norgestrel/levonorgestrel) is exceptionally proposed in the following scheme [10]:

- 1 pill every 4 hours until bleeding stops or is significantly reduced then:

- 1 pill 4 times/day for 2-4 days;

- 1 pill 3 times/day for 3 days;

- 1 pill 2 times/day until the end of the 21-day treatment cycle.

With such high doses of OCs, it is usually necessary to include antiemetics. OCs therapy should be continued ( 1 tablet/day) for the next 3-6 menstrual cycles. OCs should be used in the $21+7$ scheme, but if the withdrawal bleed 
is too heavy, the interval between consecutive packages should be shortened to 3-4 days [10].

When severe AUB $(\mathrm{Hb}<7 \mathrm{~g} / \mathrm{dL})$ is accompanied by symptoms of haemodynamic failure, urgent hospitalization is recommended; the necessity to transfuse fluids and red blood cells preparation (individual indications) should also be taken into account. In the case of very massive bleeding, intravenous/intramuscular administration of estrogens is recommended to obtain a hemostatic effect: $25 \mathrm{mg}$ of conjugated estrogens iv. every 4-6 hours or 10-20 mg i.m. estradiol valerate once (Estradiol Depot) during the first day. OCs should be turned on in the next 24-48 hours to deliver the progestogen and stabilize the endometrium; then use OCs continuously for several consecutive cycles, abandoning menstrual bleeding in order to compensate for iron deficiency [1-10].

In case of contraindications to estrogen therapy or OCs, progestogens can be used (MPA $10 \mathrm{mg}$, NETA 5-10 mg or lynesterol 5-10 mg 4 times/day for 4 days, 3 times/day for 3 days and then 2 times/day for 14 days) but the effectiveness of such therapy in severe AUB is limited [9].

An alternative treatment option for severe juvenile bleeding is the intrauterine hormonal contraceptive system (releasing levonorgestrel) or long-acting $\mathrm{GnRH}$ analogues (used for the longest period of 6 months) [11].

Abrasion of the uterine cavity may be considered as an exceptional indication in girls when hormonal treatment (various regimens) is ineffective and bleeding continues for another 24-36 hours [2].

In the adjunctive therapy of severe AUB, the following are recommended: antiemetics, antifibrinolytics, vitamins, iron and folic acid supplementation (anemia correction), NSAIDs and antibiotic therapy. Antibiotic therapy is recommended in order to reduce the inflammatory component in cases where bleeding lasts more than 10 days, the uterus is painful at examination, and when there are symptoms of infection [1-9].

The correct diagnostic and therapeutic process of AUB should stop the bleeding and prevent its recurrence, explain the cause of the symptoms and prevent the intensification of co-occurring endocrine disorders (e.g. acne, hirsutism, obesity in the course of PCOS). It is estimated that about
$10-15 \%$ of girls with symptoms of juvenile bleeding will forever remain anovulatory menstrual cycles and will develop PCOS. Therefore, in the case of abnormal uterine bleeding during adolescence, long-term follow-up is recommended, ensuring constant control of symptoms and preventing long-term complications interfering the reproductive functions of a young woman (persistent disorders of the menstrual cycle, hormonal dysfunction, fertility disorders).

\section{Conflict of interest}

None declared.

\section{REFERENCES}

1. Sokkary N, Dietrich JE. Management of heavy menstrual bleeding in adolescents. Curr Opin Obstet Gynecol. 2012; 24(5): 275-280, doi: 10.1097/GCO.0b013e3283562bcb, indexed in Pubmed: 22729091.

2. Haamid F, Sass AE, Dietrich JE. Heavy menstrual bleeding in adolescents. J Pediatr Adolesc Gynecol. 2017; 30(3): 335-340, doi: 10.1016/j. jpag.2017.01.002, indexed in Pubmed: 28108214.

3. Mullins TL, Miller RJ, Mullins ES. Evaluation and management of adolescents with abnormal uterine bleeding. Pediatr Ann. 2015; 44(9): e218-e222, doi: 10.3928/00904481-20150910-09, indexed in Pubmed: 26431240.

4. Committee on Practice Bulletins-Gynecology. Practice bulletin no. 128: diagnosis of abnormal uterine bleeding in reproductive-aged women. Obstet Gynecol. 2012; 120(1): 197-206, doi: 10.1097/AOG.0b013e318262e320, indexed in Pubmed: 22914421.

5. Bennett AR, Gray SH. What to do when she's bleeding through: the recognition, evaluation, and management of abnormal uterine bleeding in adolescents. Curr Opin Pediatr. 2014; 26(4): 413-419, doi: 10.1097/MOP.0000000000000121, indexed in Pubmed: 25007322.

6. Matteson KA, Scott DM, Raker CA, et al. The menstrual bleeding questionnaire: development and validation of a comprehensive patient-reported outcome instrument for heavy menstrual bleeding. BJOG. 2015; 122(5): 681-689, doi: 10.1111/1471-0528.13273, indexed in Pubmed: 25615842.

7. LaCour DE, Long DN, Perlman SE. Dysfunctional uterine bleeding in adolescent females associated with endocrine causes and medical conditions. J Pediatr Adolesc Gynecol. 2010; 23(2): 62-70, doi: 10.1016/j. jpag.2009.06.003, indexed in Pubmed: 20347757.

8. Long S. Implementing screening recommendations for adolescents with heavy menstrual bleeding. The Journal of Pediatrics. 2015; 166(1): 1-3, doi: 10.1016/j.jpeds.2014.10.061.

9. Hickey M, Higham J, Fraser IS, et al. Progestogens versus oestrogens and progestogens for irregular uterine bleeding associated with anovulation. Cochrane Database Syst Rev. 2000(2): CD001895, doi: 10.1002/14651858. CD001895, indexed in Pubmed: 10796833.

10. Farquhar C, Brown J. Oral contraceptive pill for heavy menstrual bleeding. Cochrane Database Syst Rev. 2009(4): CD000154, doi: 10.1002/14651858.CD000154.pub2, indexed in Pubmed: 19821266.

11. Lethaby $A E$, Cooke I, Rees $M$, et al. Progesterone/progestogen releasing intrauterine systems versus either placebo or any other medication for heavy menstrual bleeding. Cochrane Database Syst Rev. 2000(2): CD002126, doi: 10.1002/14651858.CD002126, indexed in Pubmed: 10796865. 